Electronic structure and thermoelectric properties of bismuth telluride and bismuth selenide

This article has been downloaded from IOPscience. Please scroll down to see the full text article.

1997 J. Phys.: Condens. Matter 9461

(http://iopscience.iop.org/0953-8984/9/2/014)

View the table of contents for this issue, or go to the journal homepage for more

Download details:

IP Address: 128.206.162.204

The article was downloaded on $23 / 12 / 2010$ at $15: 38$

Please note that terms and conditions apply. 


\title{
Electronic structure and thermoelectric properties of bismuth telluride and bismuth selenide
}

\author{
S K Mishra $\ddagger$, S Satpathy $\ddagger$ and O Jepsen $\ddagger$ \\ $\dagger$ Department of Physics \& Astronomy, University of Missouri, Columbia, MO 65211, USA \\ $\ddagger$ Max-Planck-Institut für Festkörperforschung, D-70569 Stuttgart, Germany
}

Received 19 June 1996, in final form 18 October 1996

\begin{abstract}
The electronic structures of the two thermoelectric materials $\mathrm{Bi}_{2} \mathrm{Te}_{3}$ and $\mathrm{Bi}_{2} \mathrm{Se}_{3}$ are studied using density-functional theory with the spin-orbit interaction included. The electron states in the gap region and the chemical bonding can be described in terms of $\mathrm{pp} \sigma$ interaction between the atomic $\mathrm{p}$ orbitals within the 'quintuple' layer. $\mathrm{For} \mathrm{Bi}_{2} \mathrm{Se}_{3}$, we find both the valenceband maximum as well as the conduction-band minimum, each with a nearly isotropic effective mass, to occur at the zone centre in agreement with experimental results. $\mathrm{For}_{\mathrm{Bi}} \mathrm{Te}_{3}$, we find that the six valleys for the valence-band maximum are located in the mirror planes of the Brillouin zone and they have a highly anisotropic effective mass, leading to an agreement between the de Haas-van Alphen data for the p-doped samples and the calculated Fermi surface. The calculated conduction band, however, has only two minima, instead of the six minima indicated from earlier experiments. The calculated Seebeck coefficients for both p-type and n-type materials are in agreement with the experiments.
\end{abstract}

Bismuth telluride and its selenide alloys are the classic thermoelectric materials with large Seebeck coefficients and, as such, they have been used in thermoelectric refrigeration for a long time $[1,2]$. There have been several attempts to fabricate better thermoelectric materials, and materials such as skutterdite pnictides and superlattices consisting of bismuthtelluride-type layers have been recently proposed [3-7]. In spite of their technological importance and the wealth of existing experimental data on $\mathrm{Bi}_{2} \mathrm{Te}_{3}$ and $\mathrm{Bi}_{2} \mathrm{Se}_{3}$, surprisingly enough, serious studies of the electronic structure of these compounds based on theoretical calculations have been started only recently.

Several early attempts [8-10] at the theoretical determination of the electronic band structure of $\mathrm{Bi}_{2} \mathrm{Te}_{3}$ relied on the empirical pseudopotential approach. To our knowledge, the only density-functional calculation for $\mathrm{Bi}_{2} \mathrm{Te}_{3}$ reported in the literature was performed by Thomas et al [11] using the LAPW method. However, in their calculation, even though the spin-orbit coupling effects were recognized to be large and significant, the spin-orbit coupling was included only empirically with a tight-binding fitting procedure, which was not adequate for describing the important details of the valence- and conduction-band valleys of these materials, which are small-band-gap semiconductors. The only band-structure calculation of the other compound, namely $\mathrm{Bi}_{2} \mathrm{Se}_{3}$, reported in the literature is an early calculation using a pseudopotential technique [12]. In this paper, we study the electronic band structures of $\mathrm{Bi}_{2} \mathrm{Te}_{3}$ and $\mathrm{Bi}_{2} \mathrm{Se}_{3}$ on the basis of density-functional calculations taking spin-orbit coupling effects into account.

The crystal structures of both $\mathrm{Bi}_{2} \mathrm{Se}_{3}$ and $\mathrm{Bi}_{2} \mathrm{Te}_{3}$ are rhombohedral with the space group $\mathrm{D}_{3 \mathrm{~d}}^{5}(R \overline{3} m)[13,14]$ with five atoms in the trigonal unit cell. The structure is most simply 

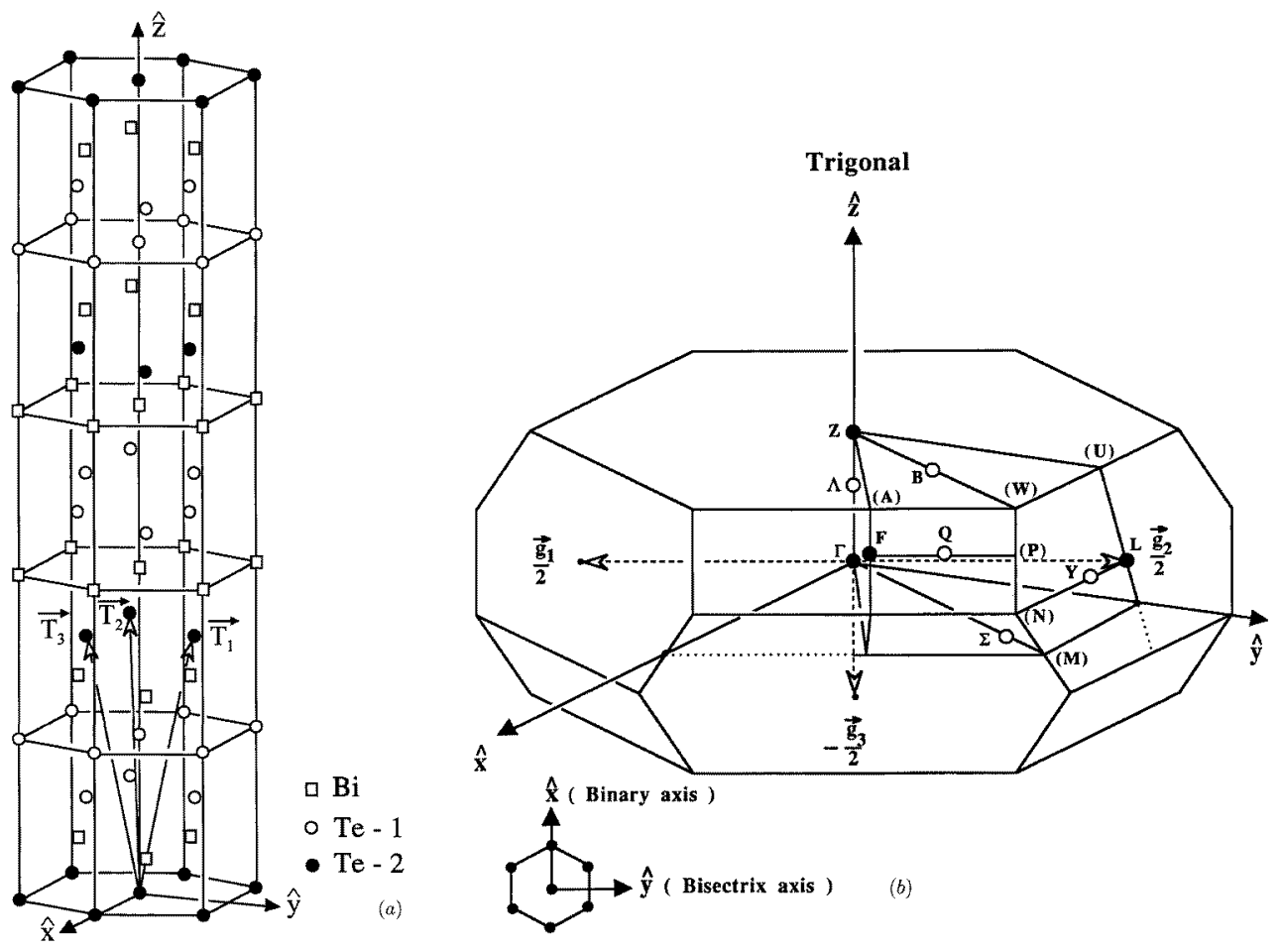

Figure 1. Crystal structures of $\mathrm{Bi}_{2} \mathrm{Se}_{3}$ and $\mathrm{Bi}_{2} \mathrm{Te}_{3}(a)$. The open and dark circles denote the $\mathrm{Te}$ and the $\mathrm{Se}$ atoms, with the $\mathrm{Bi}$ atoms shown as squares. The irreducible $\mathrm{BZ}$ is shown in (b). The points in the BZ shown in parentheses are strictly not points of symmetry. The binary axis (with twofold rotation symmetry) is along $\boldsymbol{x}$, the bisectrix axis (occurring in the reflection plane) is along $\boldsymbol{y}$, and the trigonal axis (with threefold rotation symmetry) is along $\boldsymbol{z}$.

Table 1. Atom positions and sphere sizes for the LMTO calculations for $\mathrm{Bi}_{2} \mathrm{Te}_{3}$ and $\mathrm{Bi}_{2} \mathrm{Se}_{3}$. E denotes an empty sphere.

Muffin-tin sphere radius $(\AA)$

\begin{tabular}{llll}
\cline { 3 - 3 } Atom & Site index & $\mathrm{Bi}_{2} \mathrm{Te}_{3}$ & $\mathrm{Bi}_{2} \mathrm{Se}_{3}$ \\
\hline $\mathrm{Bi}$ & $2 \mathrm{c}(x=0.399)$ & 1.78 & 1.75 \\
$\mathrm{Te} 1 / \mathrm{Se} 1$ & $2 \mathrm{c}(x=0.792)$ & 1.76 & 1.58 \\
$\mathrm{Te} 2 / \mathrm{Se} 2$ & $1 \mathrm{a}$ & 1.88 & 1.69 \\
$\mathrm{E} 1$ & $1 \mathrm{~b}$ & 1.45 & 1.44 \\
$\mathrm{E} 2$ & $2 \mathrm{c}(x=0.10)$ & 1.28 & 1.22 \\
$\mathrm{E} 3$ & $2 \mathrm{c}(x=0.30)$ & 1.26 & 1.18 \\
\hline Space group: & $\mathrm{D}_{3 \mathrm{~d}}^{5}(R \overline{3} m)$, rhombohedral unit cell & & \\
Lattice constant, $a:$ & & $10.45 \AA$ & $9.84 \AA$ \\
Rhombohedral angle, $\alpha:$ & & $24^{\circ} 8^{\prime}$ & $24^{\circ} 8^{\prime}$ \\
\hline
\end{tabular}

visualized in terms of a layer structure and a hexagonal cell sketched in figure 1 . The structure may be described as quintuple layers of atoms ('quintuple layer leaves'), which are stacked along the $c$-axis. The five individual atomic layers making up the quintuple layer occur in the sequence $\mathrm{Te}(1)-\mathrm{Bi}-\mathrm{Te}(2)-\mathrm{Bi}-\mathrm{Te}(1)$, where $\mathrm{Te}(1)$ and $\mathrm{Te}(2)$ denote the two 

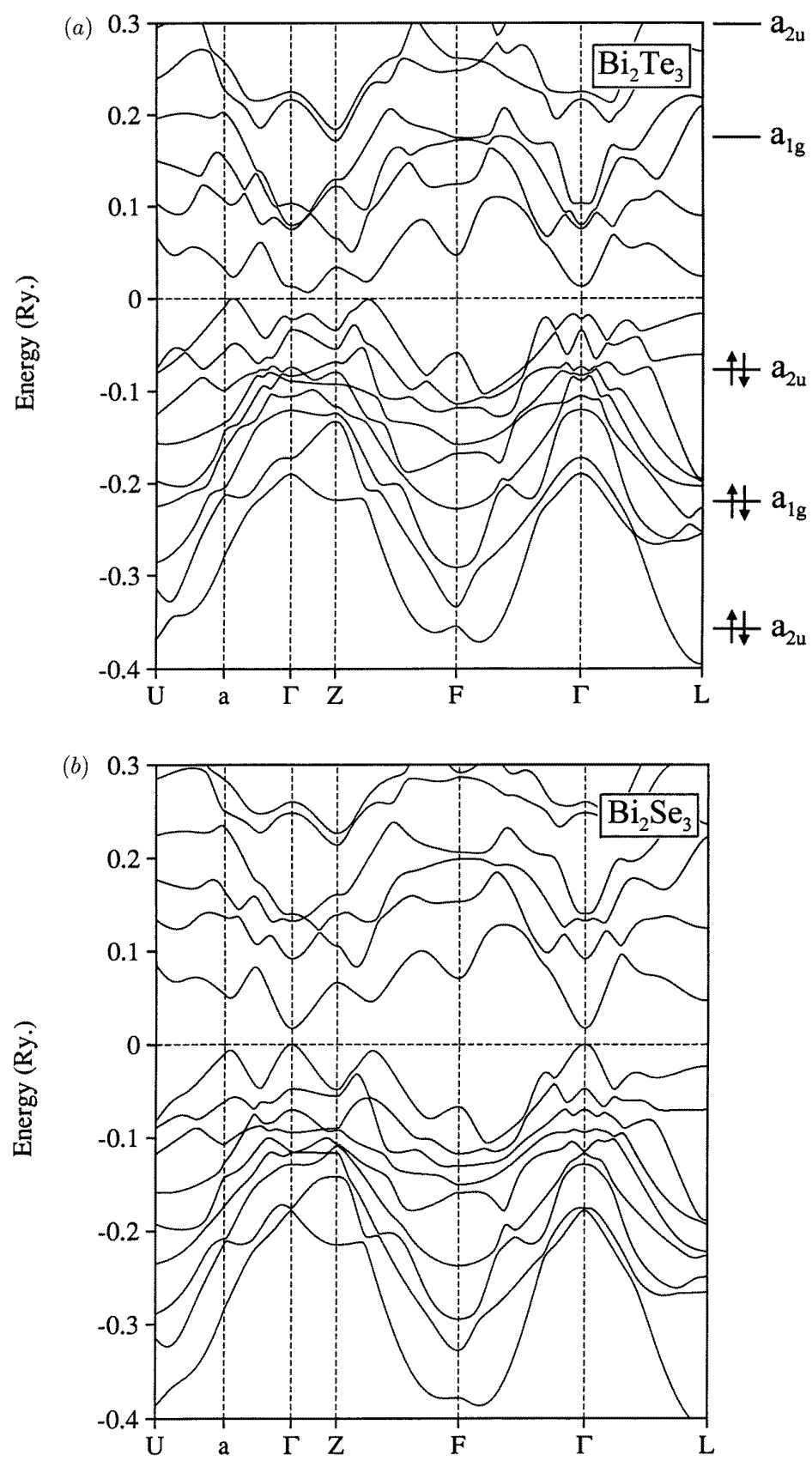

Figure 2. Electronic band structures for $\mathrm{Bi}_{2} \mathrm{Te}_{3}(a)$ and $\mathrm{Bi}_{2} \mathrm{Se}_{3}(b)$ obtained from LMTO-LDA

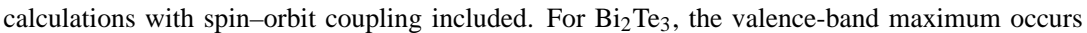
along the $\Gamma-$ a direction (the inset of figure 3 shows the location of the point ' $a$ ' in the BZ). The valence-band top along the $\mathrm{Z}-\mathrm{F}$ direction has energy comparable to the valence-band maximum

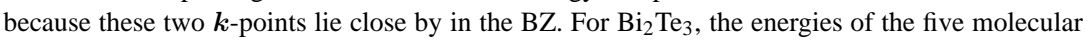
orbitals (MOs) obtained from a simple $5 \times 5$ tight-binding model are indicated. Each of the MOs produces three bands because of the threefold degeneracy of the p orbitals. 


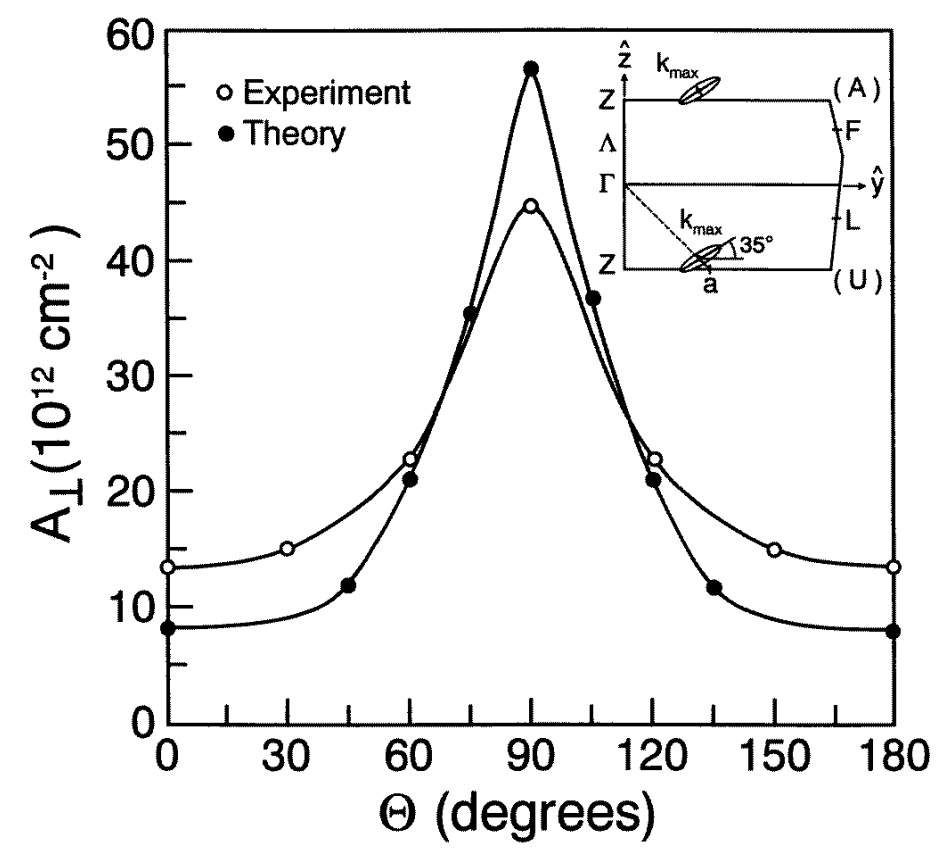

Figure 3. A comparison of the calculated extremal cross-sectional areas $A_{\perp}$ as a function of the azimuthal angle $\theta$ with the $\mathrm{dHvA}$ measurement for $\mathrm{p}$-doped $\mathrm{Bi}_{2} \mathrm{Te}_{3}$ with a carrier concentration of $4 \times 10^{18} \mathrm{~cm}^{-3}$ corresponding to a hole pocket with $E_{F}=21.8 \mathrm{meV}$. The inset shows the location of the valence-band maximum in the BZ obtained from the present calculation. Solid lines through the point $k_{\max }$ corresponding to the valence-band maximum indicate the directions of the principal axes of the mass tensor.

different types of tellurium atom in the crystal. The description in terms of the stacking of the quintuple layers is justified in view of the appreciable distances between adjacent $\mathrm{Te}(1)$ layers and it is an important structural feature for the description of the chemical bonding as discussed below. Each atom is surrounded by six atoms, three in the layer below and three in the layer above, along the $c$-axis in nearly octahedral coordination, except for the $\mathrm{Te}(1)$ atoms whose three nearest-neighbour $\mathrm{Te}(1)$ atoms, out of a total of six, are located on a different 'quintuple' layer leaf, and hence are somewhat further away from the octahedral coordination.

The band structures are calculated using the local-density approximation (LDA) to the density-functional theory with the linear muffin-tin orbital method in the atomic-sphere approximation (LMTO-ASA) [15]. The von Barth-Hedin exchange-correlation potential was used [16]. Muffin-tin orbitals of angular momenta s, $p$, and $d$ are retained on all atoms and on the several empty spheres which were introduced in the interstitial region. Tests to see whether the inclusion of orbitals with higher angular momenta (e.g., s, p, d and $f$ on all spheres) altered the states near the gap region were performed, but they did not affect the results significantly. Core electrons were treated self-consistently ('soft' cores) and with relativistic effects taken into account. The positions of the atoms and the empty spheres as well as the sphere radii used in the calculations are given in table 1.

The electron band structures for the two compounds are shown in figure 2. We find that the spin-orbit coupling introduces significant changes in the band structure for $\mathrm{Bi}_{2} \mathrm{Te}_{3}$ in 
the gap region (which is consistent with the results of Thomas et al [11]), but it has very little effect for $\mathrm{Bi}_{2} \mathrm{Se}_{3}$. In fact, for the former, without the spin-orbit coupling effects, one obtains a single-valley valence-band maximum and the same for the conduction-band minimum, both occurring at the $\Gamma$ point.

The calculated electronic band structure for $\mathrm{Bi}_{2} \mathrm{Se}_{3}$ shows a one-valley conduction-band minimum and a one-valley valence-band maximum occurring at the centre of the Brillouin zone (BZ) and we find both valleys to have an isotropic effective mass. The nature of the conduction-band minimum has been studied experimentally [20] and it is consistent with our results. The experimental data on the nature of the valence-band maximum are, however, limited because of the difficulty of making the p-doped samples. On the basis of an early galvanomagnetic measurement, a single-valley valence-band maximum located at the $\Gamma$ point was postulated [21]. This is confirmed by the present calculations. The calculated gap value is about $0.24 \mathrm{eV}$, consistent with the measured value of $0.2-0.3 \mathrm{eV}$ [22].

For $\mathrm{Bi}_{2} \mathrm{Te}_{3}$, there are six valleys for the highest valence band located in the mirror planes of the $\mathrm{BZ}$ at the point shown in the inset of figure 3, which shows the calculated location of the valence-band maximum in the BZ. The valleys are from experiments known to be located on the plane containing the trigonal and the bisectrix axis, which is consistent with our results. The effective masses are highly anisotropic. From symmetry considerations, the energy near the valence-band maximum can be written as

$$
2 m_{0} E / \hbar^{2}=\alpha_{11} k_{x}^{2}+\alpha_{22} k_{y}^{2}+\alpha_{33} k_{z}^{2}+2 \alpha_{23} k_{y} k_{z}
$$

where $k$ is the Bloch vector, $m_{0}$ is the free-electron mass, and the mass parameters $\alpha$ are related to the effective-mass tensor. Our fitted values of the mass parameters are: $\alpha_{11}=109.3, \alpha_{22}=5.2, \alpha_{33}=6.2$ and $\alpha_{23}=3.1$, which leads to the principal axes of the energy ellipsoid making an angle of about $35^{\circ}$ with the crystalline axes as shown in figure 3 . This is in very good agreement with the measured value of $31.5^{\circ} \pm 10 \%$ [17]. Figure 3 shows the satisfactory agreement between the experimental and calculated extremal crosssectional areas of one Fermi surface ellipsoid for the p-doped sample corresponding to the Fermi energy $E_{F}=21.8 \mathrm{meV}$, with the magnetic field perpendicular to the $c$-axis.

The conduction-band minimum occurs along the $\Lambda(\Gamma-\mathrm{Z})$ line in the BZ, which results in two valleys for the conduction-band minimum with a more-or-less isotropic mass tensor. This is in disagreement with the experiment which shows six valleys located on a mirror plane of the BZ [18]. We note that the $\Lambda$ line is in fact on the mirror plane of the BZ, but because we have additional symmetry for the $\Lambda$ line, only two valleys occur if the minimum is located on this line. One would get six valleys if this minimum was slightly displaced from the $\Lambda$ line. A small change in the electronic band structure, resulting perhaps from the inclusion of the non-spherical part of the potential (omitted in the present atomic-sphere approximation), may be sufficient to explain the observed six-valley minimum. Within the ASA, we have considered small changes in the atomic positions of the crystal, which resulted in the same valley structure. The calculated band gap is about $0.11 \mathrm{eV}$ for $\mathrm{Bi}_{2} \mathrm{Te}_{3}$ as compared to the experimental value of $0.15 \mathrm{eV}$ [19].

The picture of chemical bonding can be understood in the following way. First of all, we note from the partial density of states (DOS) (figure 4) that the valence and conduction bands consist of the metal and chalcogen p orbitals, while the bands arising from the valence $\mathrm{s}$ orbitals occur about 0.8 Ryd below the valence-band maximum, consistent with the photoemission experiments [23]. Focusing on $\mathrm{Bi}_{2} \mathrm{Te}_{3}$, the electron states in the vicinity of the gap region consist of the $\mathrm{Bi}(\mathrm{p})$ and the $\mathrm{Te}(\mathrm{p})$ orbitals, since all other orbitals lie far away in energy [24]. As discussed already, the structure consists of quintuple layers stacked on top of one another, with the layer sequence of $\mathrm{Te}(1)-\mathrm{Bi}-\mathrm{Te}(2)-\mathrm{Bi}-\mathrm{Te}(1)$ within 


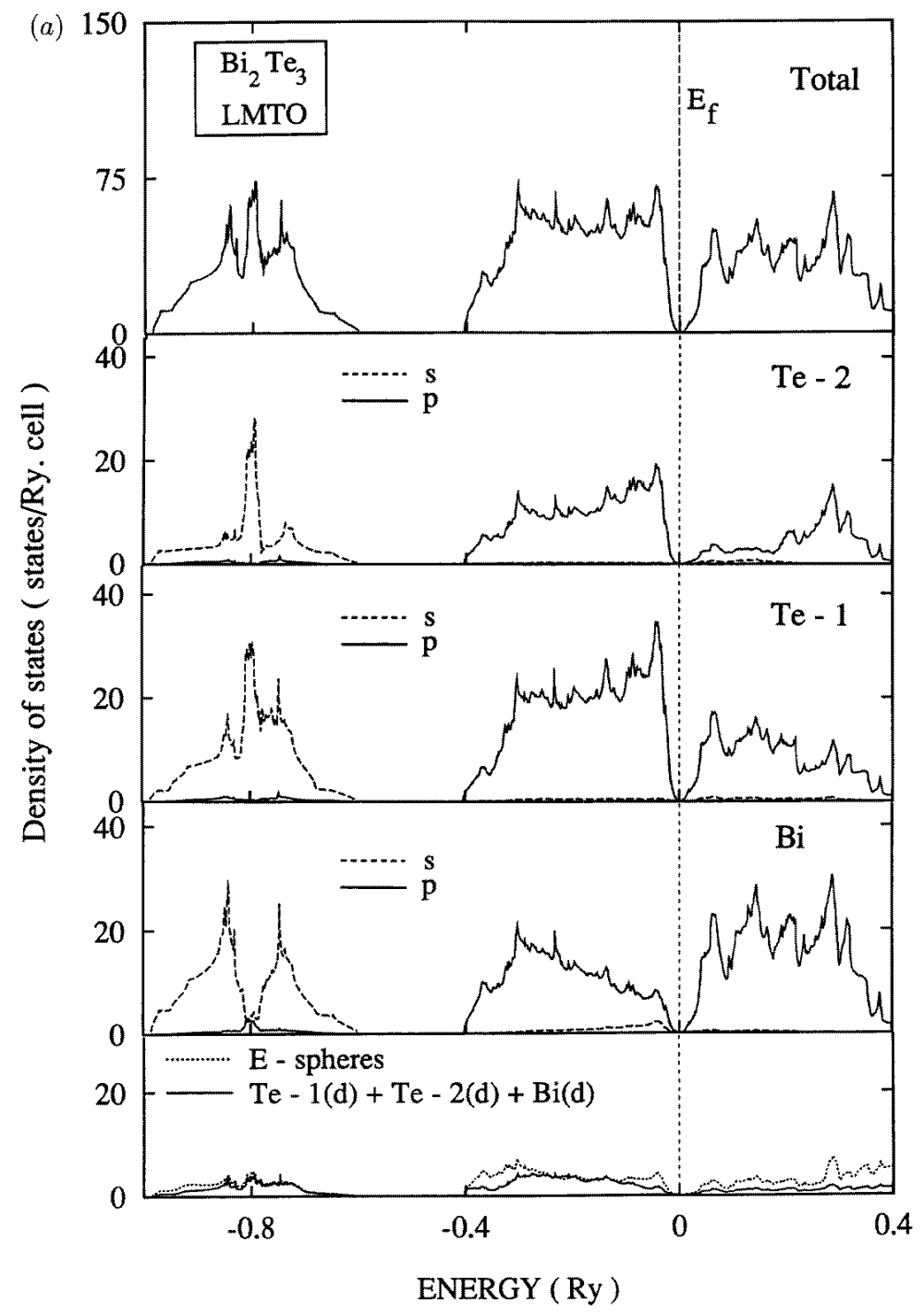

Figure 4. One-electron densities of states for $(a) \mathrm{Bi}_{2} \mathrm{Te}_{3}$ and $(b) \mathrm{Bi}_{2} \mathrm{Se}_{3}$ with spin-orbit coupling included.

each quintuple layer. Each of the three p orbitals on an atom points towards an atom on the layer above, and one on the layer below, in such a way that, since the five atoms lie roughly on a straight line, we get a sequence of five p orbitals interacting via the $\mathrm{pp} \sigma$ interaction. (See figure 5.)

If we now keep the predominant $\mathrm{pp} \sigma$ interaction between nearest-neighbour atoms and only within a quintuple layer, then the electron states can be described by a $5 \times 5$ tight-binding Hamiltonian matrix. There are then two parameters entering the tight-binding Hamiltonian, namely the difference of the on-site $\mathrm{p}$ energies and the $\mathrm{NN} p \mathrm{p} \sigma$ matrix element, which describes the Hamiltonian. Following Harrison [25], we take the various parameters to be: $\varepsilon_{p}(\mathrm{Bi})=-7 \mathrm{eV}, \varepsilon_{p}(\mathrm{Te})=-8.5 \mathrm{eV}$, and $V_{\mathrm{pp} \sigma}=-2.5 \mathrm{eV}$, which then results in the 


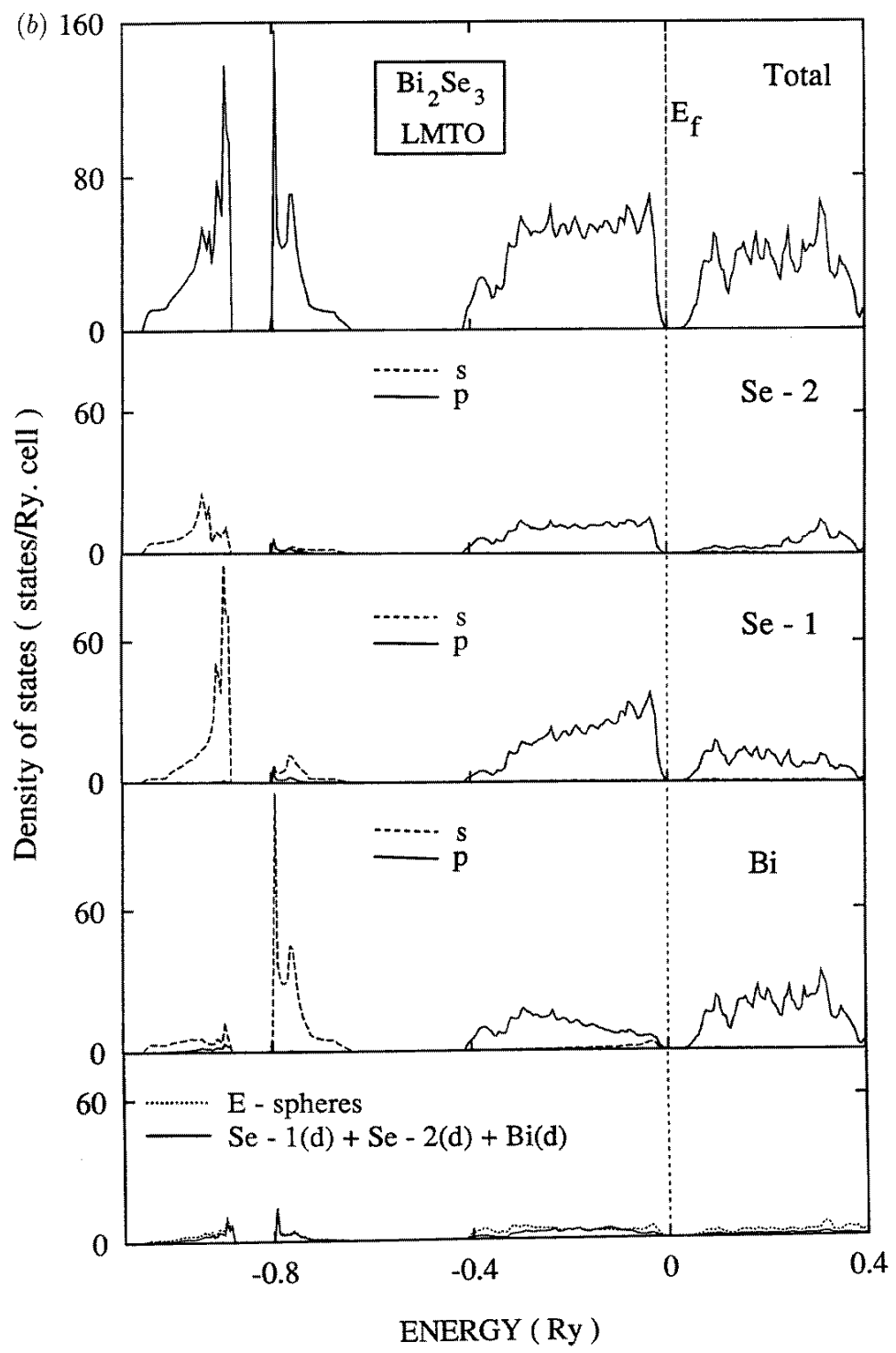

Figure 4. (Continued)

eigenvalues of $\varepsilon=-12.1,-10.4,-8.5,-5.1$, and $-3.4 \mathrm{eV}$. These are indicated along with their symmetries in figure 5 and also in the band-structure plot for $\mathrm{Bi}_{2} \mathrm{Te}_{3}$ in figure $2(a)$. The five $p$ orbitals span the $2 a_{1 g}+3 a_{2 u}$ irreducible representations of the relevant $D_{\infty h}$ point group as seen from the figure. Of these five molecular orbitals, the lowest two are bonding in character, the highest two are anti-bonding, and the remaining one is non-bonding. The valence-electron count is such that the lowest three of these orbitals are occupied, leading to the ionic charges given roughly by the chemical formula $\mathrm{Bi}_{2}^{0.7+} \mathrm{Te}(1)_{2}^{0.5-} \mathrm{Te}(2)^{0.35-}$, extracted from the eigenvectors of the $5 \times 5$ Hamiltonian. (The charges do not add up to zero because of rounding.) The charge-density contour plots (figure 6) clearly show the metal atoms to be positively charged and the chalcogen atoms to be negatively charged for both $\mathrm{Bi}_{2} \mathrm{Se}_{3}$ 


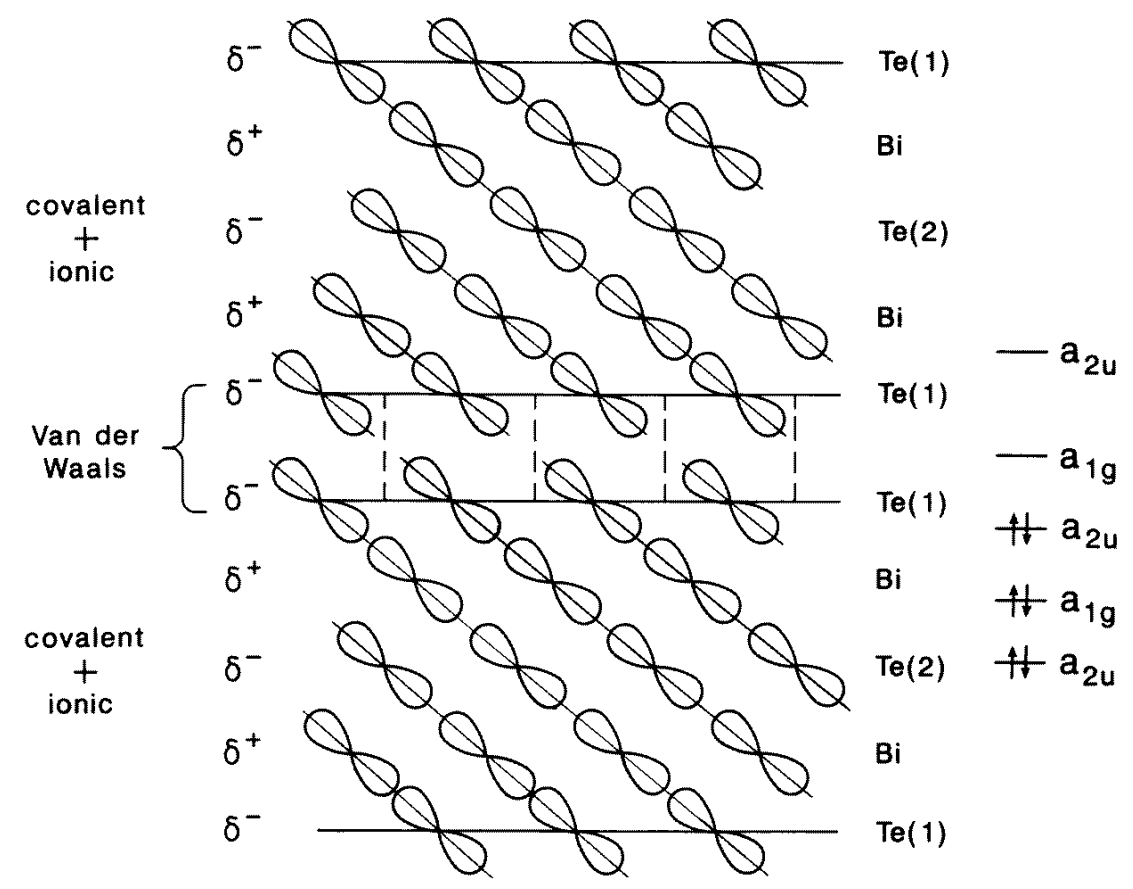

Figure 5. A sketch of the chemical bonding consistent with the results of the density-functional calculations. The molecular orbitals produced by the $\mathrm{pp} \sigma$ interaction on the five-atom linear chain are shown on the right.

and $\mathrm{Bi}_{2} \mathrm{Te}_{3}$ providing support for the simple picture of chemical bonding. Thus the bonding between the atoms within a quintuple layer is of covalent-ionic type, while the interaction between two $\mathrm{Te}(1)$ layers belonging to two different quintuple layers is of the Van der Waals type as indicated in figure 5. After we completed the analysis of the chemical bonding, we became aware of the work of Pecheur and Toussaint [26], who also proposed a picture of chemical bonding governed by the pp $\sigma$ interactions similar to ours.

The thermoelectric power or the Seebeck coefficient is given by $\alpha=(2 / 3) C_{v} / n e$ from the transport equation within the approximation where the mean free path is independent of the energy of the carrier (electron or hole). (The factor of two-thirds disappears if we make the approximation that the relaxation time $\tau$ rather than the mean free path is constant [27].) For a parabolic band, the above expression simplifies to

$$
\alpha=-(2 / 3) \pi^{2} k_{B}^{2} T /\left(2 e \epsilon_{F}\right) .
$$

The measured value of $\alpha$ at room temperature is reported [1] to be $\sim 260 \mu \mathrm{V} \mathrm{K}^{-1}$ for p-type $\mathrm{Bi}_{2} \mathrm{Te}_{3}$ with a hole concentration of $\sim 4 \times 10^{18} \mathrm{~cm}^{-3}$ and $\sim 250 \mu \mathrm{V} \mathrm{K} \mathrm{K}^{-1}$ for $\mathrm{n}$ type samples with an electron concentration of $\sim 7.4 \times 10^{18} \mathrm{~cm}^{-3}$. These compare very well with our calculated values of $313 \mu \mathrm{V} \mathrm{K}^{-1}$ and $196 \mu \mathrm{V} \mathrm{K}^{-1}$ for the p-doped and the n-doped cases, respectively, using $T=300 \mathrm{~K}$. Similarly, for $\mathrm{Bi}_{2} \mathrm{Se}_{3}$, the measured value of $\sim 10 \mu \mathrm{V} \mathrm{K}^{-1}$ for both n-type and p-type samples with carrier concentrations of $4 \times 10^{19} \mathrm{~cm}^{-3}$ and $3 \times 10^{19} \mathrm{~cm}^{-3}$, respectively, at $T=400 \mathrm{~K}$ [28], agrees reasonably well with the calculated values of $13.5 \mu \mathrm{V} \mathrm{K}^{-1}$ and $12.8 \mu \mathrm{V} \mathrm{K} \mathrm{K}^{-1}$.

In summary, using local-density calculations we have studied the electronic structure of $\mathrm{Bi}_{2} \mathrm{Se}_{3}$ and $\mathrm{Bi}_{2} \mathrm{Te}_{3}$ and have obtained a simple picture of chemical bonding in these materials. 

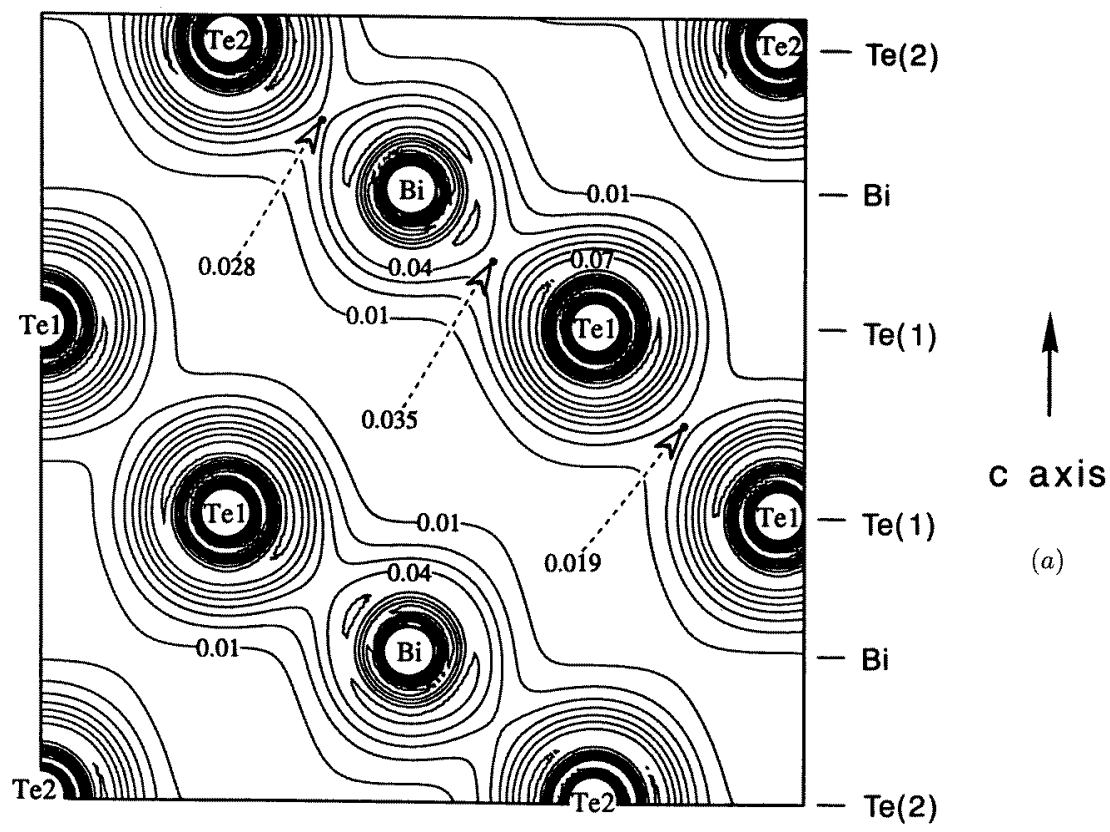

(a)

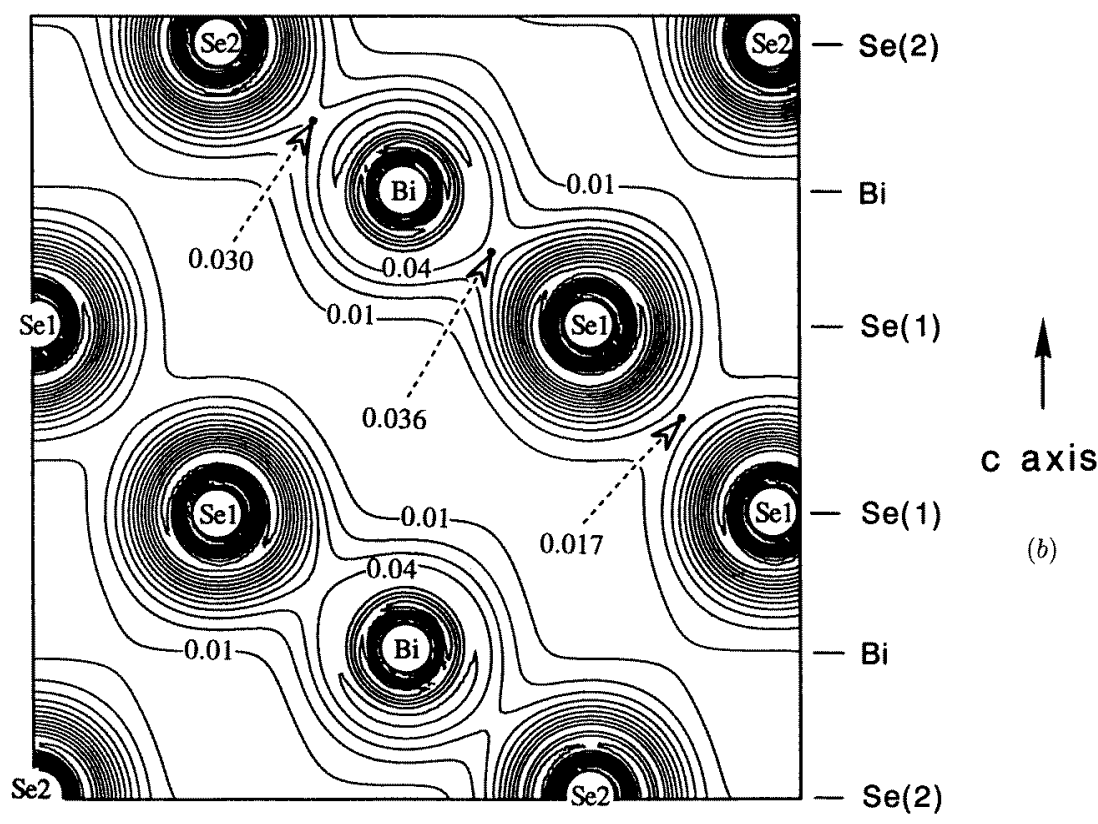

Figure 6. A valence charge-density contour plot on the $x z$-plane for $\mathrm{Bi}_{2} \mathrm{Te}_{3}(a)$. Contour values are in units of electrons $\mathrm{au}^{-3}$. The stacking of the various layers of atoms along the $c$-axis is indicated on the right. The plot supports the covalent-ionic picture of chemical bonding with the metal (Bi) atoms positively charged and the chalcogen (Te or $\mathrm{Se}$ ) atoms negatively charged. The $\mathrm{Te}(1)$ atoms, which form the outer boundary layers of the quintuple layer structure, are weakly bonded via van der Waals forces, leading to an appreciably smaller electron charge density at the centre of the $\mathrm{Te}(1)-\mathrm{Te}(1)$ bonds. The same picture of chemical bonding is supported by the charge-density contour plot for $\mathrm{Bi}_{2} \mathrm{Se}_{3}(b)$. 
The calculated thermoelectric power agrees reasonably well with the experiments for both materials.

\section{Acknowledgments}

We thank M-C Chen for valuable discussions. This work was supported in part by the Office of Naval Research under contract No ONR N00014-95-1-0439

\section{References}

[1] Goldsmid H J 1964 Thermoelectric Refrigeration (New York: Plenum)

[2] Ioffe A F 1957 Semiconductor Thermoelements and Thermoelectric Cooling (London: Infosearch)

[3] Caillat T, Borshchevsky A and Fleurial J P 1993 Proc. 11th Int. Conf. on Thermoelectrics (Arlington, TX) ed K R Rao (Arlington, TX: University of Texas at Arlington Press)

[4] Slack G A and Tsoukala V G 1994 J. Appl. Phys. 761665

[5] Singh D J and Pickett W E 1994 Phys. Rev. B 5011235

[6] Sales B C, Mandrus D and Williams R K 1996 Science 2721325

[7] Hicks L D and Dresselhaus M S 1993 Phys. Rev. B 4712727

[8] Katsuki S I 1969 J. Phys. Soc. Japan 2658

[9] Togei R and Miller G R 1971 J. Phys. Chem. Solids Suppl. 132349

[10] Oleshko E V and Korolyshin V N 1985 Sov. Phys.-Solid State 271723

[11] Thomas G A, Rapkine D H, van Dover R B, Mattheiss L F, Sunder W A, Schneemeyer L F and Waszczak J V 1992 Phys. Rev. B 461553

[12] Oleshko E V and Korolyshin V N 1985 Sov. Phys.-Semicond. 191130

[13] Wyckoff R W G 1986 Crystal Structures vol 2 (Malabar, FL: Krieger) and references therein

[14] Wiese J R and Muldawer L 1960 J. Phys. Chem. Solids 1513

[15] Andersen O K 1975 Phys. Rev. B 123060

Skriver H L 1983 The LMTO Method (New York: Springer)

Andersen O K, Jepsen O and Glötzel D 1985 Highlights in Condensed Matter Theory ed F Bassani, F Fumi and N Tosi (Amsterdam: North-Holland)

[16] von Barth U and Hedin L 1972 J. Phys. C: Solid State Phys. 51629

[17] Köhler H 1976 Phys. Status Solidi b 74591

[18] Köhler H 1976 Phys. Status Solidi b 7395

[19] Gol'tsman B M, Kudinov V A and Smirnov I A 1972 Semiconductor Thermoelectric Materials Based on $\mathrm{Bi}_{2} \mathrm{Te}_{3}$ (Moscow: Nauka) (in Russian)

[20] Hyde G R, Beale H A, Spain I L and Woollam J A 1974 J. Phys. Chem. Solids 351719

Köhler H 1973 Phys. Status Solidi b 5891

Köhler H and Fischer H 1975 Phys. Status Solidi b 69349

[21] Köhler H and Fabricius A 1975 Phys. Status Solidi b 71487

[22] Davidenko V A 1941 J. Phys. (USSR) 4170

Mooser E and Pearson W B 1956 Phys. Rev. 101492

Black J, Conwell E M, Seigle L and Spencer C W 1957 J. Phys. Chem. Solids 2240

Konorov P P 1956 Zh. Tekh. Fiz. 26394

[23] Thuler M R, Benbow R L and Hurych Z 1982 Chem. Phys. 71265

[24] Hermann F and Skillman S 1963 Atomic Structure Calculations (Englewood Cliffs, NJ: Prentice-Hall)

[25] Harrison W A 1979 Electronic Structure and the Properties of Solids (San Francisco, CA: Freeman)

[26] Pecheur P and Toussaint G 1989 Phys. Lett. 135A 223

[27] MacDonald D K C 1962 Thermoelectricity (New York: Wiley) p 25

[28] Boechko V F and Isarev V I 1975 Inorg. Mater. 111288 